Pathophysiology

of Haemostasis and Thrombosis
Pathophysiol Haemost Thromb 2007-08;36:217-226

DOI: $\underline{10.1159 / 000252816}$
Received: May 18, 2009

Accepted after revision: July 22, 2009

\title{
Oral Factor Xa Inhibitors for Venous Thromboembolism Prevention in Major Orthopedic Surgery: A Review
}

\author{
Davide Imberti ${ }^{a}$ Domenico Prisco $^{b}$

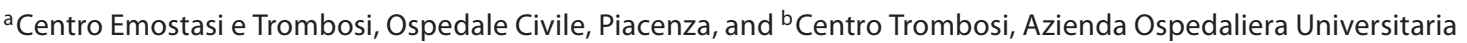 \\ Careggi, Firenze, Italy
}

\section{Key Words}

Venous thromboembolism • Factor Xa inhibitors, oral •

Orthopedic surgery, major

\begin{abstract}
Venous thromboembolism (VTE), which includes deep vein thrombosis and pulmonary embolism, is a major cause of morbidity and mortality in patients undergoing major orthopedic surgery, and routine thromboprophylaxis has been the standard of care over the last 20 years. Currently available options for the prevention of VTE in major orthopedic surgery include low-molecular-weight heparins, vitamin $\mathrm{K}$ antagonists and, more recently, the synthetic pentasaccharide fondaparinux. Although effective, these drugs have several limitations, and new oral antithrombotics offering predictable, effective and safe anticoagulation are strongly needed. This overview focuses on the most advanced oral direct inhibitors of factor Xa, rivaroxaban, apixaban, LY517717, YM150 and betrixaban. Specifically, the results of phase II and III studies and the designs of ongoing clinical trials in patients undergoing elective hip and knee replacement are reviewed.

Copyright $\odot 2009$ S. Karger AG, Basel
\end{abstract}

\section{Introduction}

Patients undergoing major orthopedic surgery, which includes total hip replacement (THR) and total knee replacement (TKR), have a particularly high risk for venous thromboembolism (VTE), and routine thromboprophylaxis has been the standard of care over the last 20 years [1-4]. Several trials have demonstrated that the rates of venographic total deep vein thrombosis (DVT) and proximal DVT following major orthopedic surgery in patients who did not undergo thromboprophylaxis are $41-85$ and $5-36 \%$, respectively, with a rate of pulmonary embolism (PE) ranging from 0.9 to $10 \%$ [4]. The introduction of routine thromboprophylaxis in these patients has reduced the overall rate of VTE, although the risk of proximal DVT is still 5-7\% and symptomatic VTE continues to be reported in $1.3-10 \%$ of patients within 3 months after surgery $[5,6]$.

Currently available anticoagulants include both parenteral and oral agents [7]. Low-molecular-weight heparins (LMWHs) have now replaced unfractionated heparin because they are more convenient to administer, and meta-analyses of clinical trials comparing LMWHs with unfractionated heparin indicate that they are more effective and safe [8]. More recently, fondaparinux, a synthet- 
Fig. 1. Indirect and direct factor Xa inhibitors.

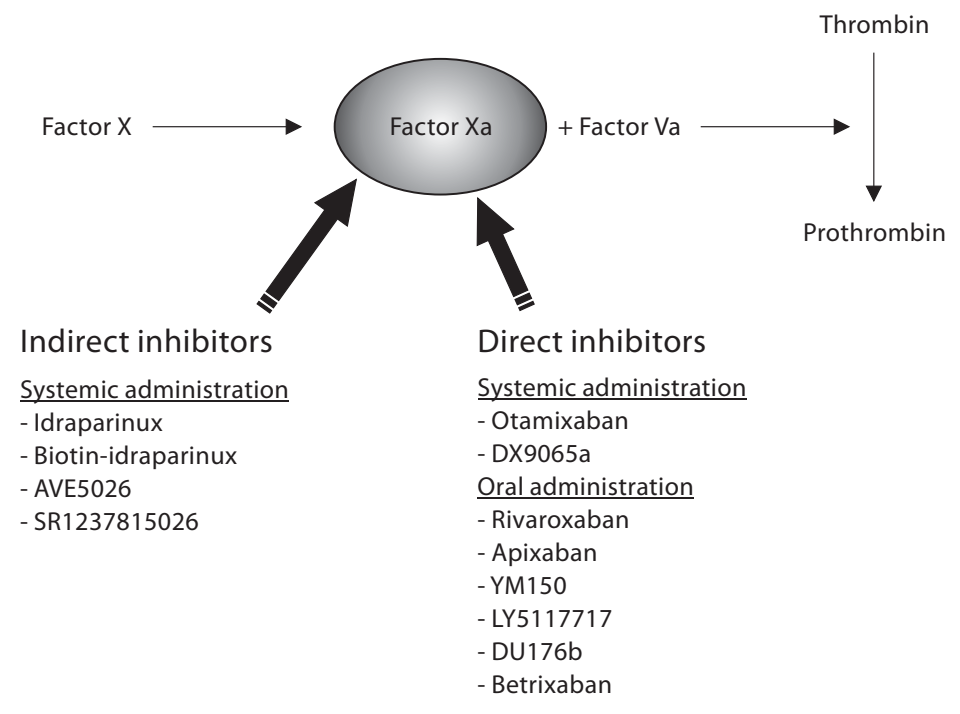

ic indirect inhibitor of factor Xa, was licensed for VTE prevention in major orthopedic surgery. In this setting fondaparinux seems to be more effective and similarly safe when compared to LMWHs [9]; conversely, both drugs can be given only by injection and may be cumbersome to use, especially in the outpatient setting. However, both unfractionated heparin and $\mathrm{LMWH}$ use is related to thrombocytopenia in $1-3 \%$ of patients, which is in turn associated with an increased risk of venous and arterial thrombosis [10]. To date, the vitamin $\mathrm{K}$ antagonists are the oral anticoagulants most frequently used for daily clinical practice. However, these drugs have certain limitations, such as a narrow therapeutic window and interaction with other drugs and food, and require regular coagulation monitoring and dose titration [11].

It is clear that a more effective and convenient alternative to these traditional methods of anticoagulation is needed. A number of new oral antithrombotic therapies targeting different steps in the coagulation cascade have been investigated, including the direct thrombin (factor IIa) inhibitors and the selective factor Xa inhibitors [12].

The inhibition of factor $\mathrm{Xa}$, and in particular its direct inhibition, seems to satisfy the still unmet need of an oral, safe and easily manageable anticoagulant drug (fig. 1) [13]. The rationale of the direct inhibition of factor Xa has recently been reviewed [14]: (1) Factor Xa is common to the intrinsic and the extrinsic activation pathways, its inhibition providing a more effective anticoagulation. (2) Factor $\mathrm{Xa}$ is the primary site of amplification, as one molecule gives origin to approximately 1,000 molecules of thrombin. (3) The functions of factor Xa seem to be restricted to promoting coagulation and inflammation.

Finally, small molecules of direct factor Xa inhibitors are able to inhibit both free and prothrombinase-bound factor Xa, and eventually even clot-associated factor Xa. This prevents all forms of factor Xa from activating prothrombin, thus contributing to counteracting the procoagulant activity of thrombi and the propagation of thrombosis.

The aim of the present review is to focus on the data of the most promising and interesting oral inhibitors of factor Xa for the prevention of VTE in major orthopedic surgery: rivaroxaban, apixaban, LY517717, YM150 and betrixaban. The most important pharmacologic characteristics of these anticoagulants are shown in table 1.

\section{Rivaroxaban}

Among the drugs of this new class, rivaroxaban (BAY 59-7939) is to date the one with the highest number of published studies, including three large phase III studies. Moreover, rivaroxaban has recently received the approval for clinical use for the prevention of VTE in major orthopedic surgery.

Phase I clinical studies showed that rivaroxaban is well tolerated in healthy human subjects, with a predictable, dose-proportional pharmacokinetic and pharmacodynamic profile $[15,16]$. It is absorbed in the gastrointestinal tract, is primarily renally excreted and has a bioavail- 
Table 1. Oral Xa inhibitors: main pharmacologic characteristics

\begin{tabular}{lllll}
\hline & Rivaroxaban & Apixaban & LY517717 & YM150 \\
\hline Ki for human factor Xa, nM & 0.4 & 0.08 & $5-7$ & 31 \\
Bioavailability, \% & $60-80$ & $50-85$ & $25-82$ & - \\
Plasma peak, $\mathrm{h}$ & $2.5-4$ & 3 & $0.5-4$ & - \\
Elimination route & $65 \%$ renal & $23 \%$ renal & primarily gastrointestinal & - \\
Half-life, h & $5-9(11-13$ in elderly) & $9-14$ & 25 & - \\
\hline
\end{tabular}

ability of more than $80 \%$ [17-21]. Plasma levels of the drug peak after 3-4 h, with a half-life ranging from 5 to $9 \mathrm{~h}$ in young individuals and from 11 to $13 \mathrm{~h}$ in the elderly [22]. Like other direct factor Xa inhibitors, rivaroxaban prolongs prothrombin time and activated partial thromboplastin time; however, the effect of the drug on these tests is short-lived, with prolongation only seen at peak drug levels.

Some drug interactions have been described with rivaroxaban [21, 22]: (1) The coadministration of gastric $\mathrm{pH}$-altering agents (antacid or ranitidine) did not influence the absorption of rivaroxaban. (2) The coadministration of aspirin $(100 \mathrm{mg})$ or the NSAID naproxen (500 $\mathrm{mg}$ ) did not alter the pharmacokinetic profile of rivaroxaban. (3) Rivaroxaban and digoxin can safely be coadministered. (4) Caution must be exercised in patients receiving treatment with rivaroxaban and potent inhibitors of both CYP3A4 and P-glycoprotein, such as ketoconazole or ritonavir.

To evaluate the efficacy and the safety of rivaroxaban in major orthopedic surgery, four phase II studies were carried out. Two studies evaluated the efficacy and safety of a twice daily regimen of rivaroxaban for 5-9 days for the prevention of VTE in patients undergoing THR or TKR. Both studies investigated the total daily dose of rivaroxaban of 5-60 $\mathrm{mg}$ and demonstrated that a wide dose range (4-fold) of 5-20 mg compared favorably with enoxaparin $[23,24]$. A third study demonstrated proof of principle for both twice and once daily rivaroxaban dosing [25], while the fourth study showed that rivaroxaban $10 \mathrm{mg}$ once daily had the optimal combination of safety and efficacy, when compared to enoxaparin $40 \mathrm{mg}$ [26]. This finding was confirmed by a pooled population analysis of phase II studies, supporting the selection of rivaroxaban $10 \mathrm{mg}$ once daily for phase III studies [27].

Four phase III trials have been conducted to evaluate the efficacy and the safety of rivaroxaban in the prophylaxis of VTE in major orthopedic surgery of the lower
Table 2. Design of the RECORD studies

\begin{tabular}{lll}
\hline Study & $\begin{array}{l}\text { Duration of } \\
\text { rivaroxaban } \\
\text { therapy }\end{array}$ & $\begin{array}{l}\text { Dose and duration of } \\
\text { enoxaparin therapy }\end{array}$ \\
\hline RECORD 1 THR & 5 weeks & 40 mg o.d.; 5 weeks \\
RECORD 2 THR & 5 weeks & 40 mg o.d.; 2 weeks \\
RECORD 3 TKR & 2 weeks & 40 mg o.d.; 2 weeks \\
RECORD 4 TKR & 2 weeks & 30 mg b.i.d.; 2 weeks \\
\hline
\end{tabular}

Rivaroxaban $10 \mathrm{mg}$ once daily (starting 6-8 h postoperatively) was compared with enoxaparin in 12,729 patients worldwide.

extremities. They have been called RECORD studies, which is the acronym for Regulation of Coagulation in Orthopedic Surgery to Prevent Deep Venous Thrombosis and Pulmonary Embolism [28-31].

More than 12,500 patients were enrolled in these four phase III multicenter, double-blind, randomized controlled trials to compare rivaroxaban and enoxaparin at different doses and treatment durations (table 2). During treatment and follow-up, laboratory variables, including liver enzymes, and cardiovascular events were monitored. The day after the last study drug dose, patients underwent mandatory bilateral venography (or earlier if the patient was symptomatic for DVT), and had a follow-up visit 30-35 days after the last dose of study drug [2831].

The trials had almost the same outcome measures: (1) The primary efficacy outcome was the composite of any DVT, nonfatal PE, or death from any cause up to 30-42 days after surgery $[28,29]$ or within 13-17 days after surgery $[30,31]$. (2) The main secondary efficacy outcome was major VTE, which was defined as the composite of proximal DVT, nonfatal PE, or death from VTE. (3) Other efficacy outcomes included the incidence of DVT (any 


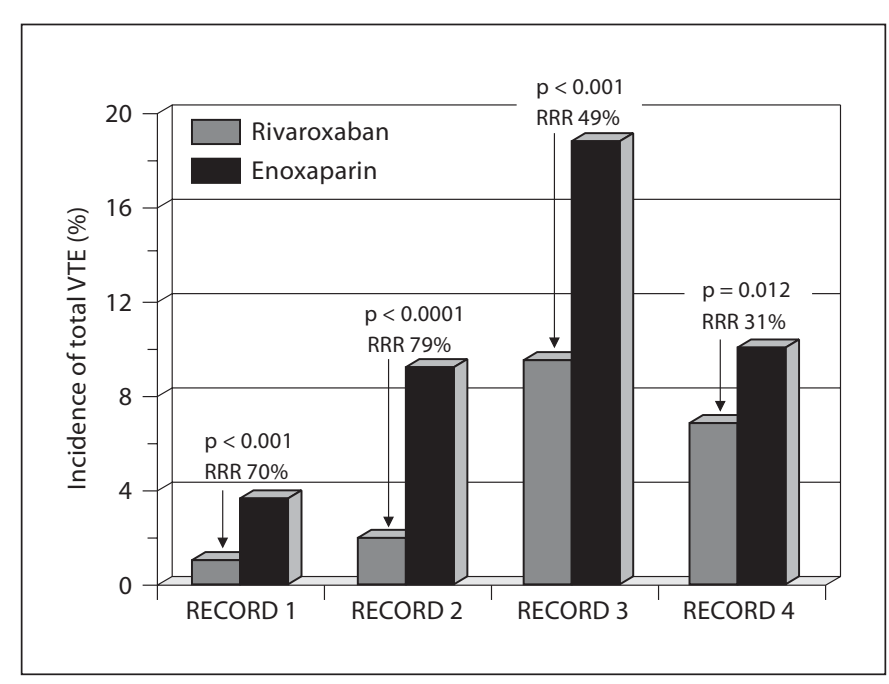

Fig. 2. Primary efficacy endpoint (composite of any DVT, nonfatal PE, or death from any cause up to 30-42 days after surgery [28, 29] or within 13-17 days after surgery $[30,31])$ in the RECORD studies. $\mathrm{RRR}=$ Relative risk reduction.

thrombosis, including both proximal and distal), the incidence of symptomatic VTE during treatment and follow-up, and death during the follow-up period.

The main safety outcome was the incidence of major bleeding beginning after the first dose of the study drug and up to 2 days after the last dose of the study drug (ontreatment period). Major bleeding was defined as bleeding that was fatal, occurred in a critical organ (e.g., retroperitoneal, intracranial, intraocular, and intraspinal bleeding), or required reoperation or extrasurgical-site bleeding that was clinically overt and associated with a fall in the hemoglobin level of at least $2 \mathrm{~g} / \mathrm{dl}$ or that required transfusion of two or more units of whole blood or packed cells. Other safety outcomes included any ontreatment bleeding, any on-treatment nonmajor bleeding, hemorrhagic wound complications (a composite of excessive wound hematoma and reported surgical-site bleeding), any bleeding that started after the first oral dose of rivaroxaban or placebo and ended up to 2 days after the last dose had been administered, adverse events, and death.

The aim of the RECORD 1, 3 and 4 studies was first to demonstrate that the efficacy of rivaroxaban was not inferior to that of enoxaparin in the per-protocol population and, if so, to determine whether rivaroxaban had superior efficacy to enoxaparin in the modified intention-to-threat population. The aim of the RECORD 2 tri- al was to determine whether 5 weeks prophylaxis with rivaroxaban was better than 2 weeks prophylaxis followed by placebo, assessed in a modified intention-tothreat population. The results presented herein are derived from the modified intention-to-treat population and from the safety population.

\section{RECORD 1 Trial [28]}

In this study, 4,541 patients undergoing elective THR were assigned to receive oral rivaroxaban $(10 \mathrm{mg}$ once daily, $\mathrm{n}=2$,266) for 35 days beginning $6-8 \mathrm{~h}$ after wound closure or subcutaneous enoxaparin (40 $\mathrm{mg}$ once daily, $\mathrm{n}=2,275)$ starting $12 \mathrm{~h}$ before surgery and restarting $6-8 \mathrm{~h}$ after wound closure. Patients also received placebo tablets or injections.

The primary efficacy outcome occurred in 18 of 1,595 patients (1.1\%) in the rivaroxaban group and in 58 of 1,558 patients (3.7\%) in the enoxaparin group, with a difference statistically significant in favor of rivaroxaban $(\mathrm{p}<0.001)$ (fig. 2). Major VTE occurred in 4 of 1,686 patients (0.2\%) in the rivaroxaban group and in 33 of 1,678 patients $(2.0 \%)$ in the enoxaparin group $(\mathrm{p}<0.001)$. This analysis showed the superiority of rivaroxaban as compared with enoxaparin. The observed rates of symptomatic VTE among patients included in the safety analysis were similar in the rivaroxaban group and the enoxaparin group ( 0.3 and $0.5 \%$, respectively).

During the treatment period, there were 4 deaths in each group in the safety population (0.2\%). On the basis of adjudication, in the rivaroxaban group, 2 deaths were possibly related to VTE, and 2 deaths were unrelated to VTE; in the enoxaparin group, 1 death was related to VTE, and 3 deaths were unrelated to VTE.

During the follow-up period, in the rivaroxaban group, 1 patient had symptomatic proximal DVT and 1 patient died from causes unrelated to VTE; in the enoxaparin group, 3 patients had symptomatic proximal DVT and 1 patient had distal DVT. During the follow-up period, there were 8 cardiovascular events in the rivaroxaban group ( 2 deaths, 2 ischemic strokes and 4 myocardial infarctions) and 1 cardiovascular death in the enoxaparin group (table 2). An on-treatment increase in the plasma alanine aminotransferase level (i.e., a level of more than tree times the upper limit of the normal range) occurred in $2.0 \%$ in the rivaroxaban group and in $2.7 \%$ in the enoxaparin group, with all cases resolving by the end of the follow-up period.

The rate of major bleeding, the combined incidence of major and clinically relevant nonmajor bleeding, the requirement for drainage and blood transfusions and the 
Table 3. Safety outcomes of the RECORD studies

\begin{tabular}{|c|c|c|c|c|c|c|c|c|}
\hline & \multicolumn{2}{|l|}{ RECORD 1} & \multicolumn{2}{|l|}{ RECORD 2} & \multicolumn{2}{|l|}{ RECORD 3} & \multicolumn{2}{|l|}{ RECORD 4} \\
\hline & $\begin{array}{l}\text { rivaroxaban } \\
(\mathrm{n}=2,209)\end{array}$ & $\begin{array}{l}\text { enoxaparin } \\
(n=2,224)\end{array}$ & $\begin{array}{l}\text { rivaroxaban } \\
(\mathrm{n}=1,228)\end{array}$ & $\begin{array}{l}\text { enoxaparin } \\
(\mathrm{n}=1,229)\end{array}$ & $\begin{array}{l}\text { rivaroxaban } \\
(\mathrm{n}=1,220)\end{array}$ & $\begin{array}{l}\text { enoxaparin } \\
(\mathrm{n}=1,239)\end{array}$ & $\begin{array}{l}\text { rivaroxaban } \\
(\mathrm{n}=1,508)\end{array}$ & $\begin{array}{l}\text { enoxaparin } \\
(\mathrm{n}=1,526)\end{array}$ \\
\hline Any on-treatment bleeding & $133(6.0)$ & $131(5.9)$ & $81(6.6)$ & $68(5.5)$ & $60(4.9)$ & $60(4.2)$ & $160(10.5)$ & $142(9.4)$ \\
\hline Major bleeding & $6(0.3)$ & $2(0.1)$ & $1(<0.1)$ & $1(<0.1)$ & $7(0.6)$ & $6(0.5)$ & $10(0.7)$ & $4(0.3)$ \\
\hline Nonmajor bleeding & $128(5.8)$ & $129(5.8)$ & $80(6.5)$ & $67(5.5)$ & $53(4.3)$ & $54(4.4)$ & $155(10.2)$ & $138(9.2)$ \\
\hline Clinically relevant nonmajor bleeding & $65(2.9)$ & $54(2.4)$ & $40(3.3)$ & $33(2.7)$ & $33(2.7)$ & $28(2.3)$ & $39(2.6)$ & $30(2.0)$ \\
\hline Hemorrhagic wound complications & $34(1.5)$ & $38(1.7)$ & $20(1.6)$ & $21(1.7)$ & $25(2.0)$ & $24(1.9)$ & NA & NA \\
\hline Patients receiving blood transfusion & $1,210(54.8)$ & $1,249(56.2)$ & $485(39.5)$ & $514(41.8)$ & $619(50.7)$ & $575(46.4)$ & NA & NA \\
\hline Drug-related adverse events & $270(12.2)$ & $265(11.9)$ & $245(20)$ & $249(20.3)$ & $146(12.0)$ & $161(13.0)$ & NA & NA \\
\hline Cardiovascular adverse events ${ }^{1}$ & $5(0.2)$ & $9(0.4)$ & $8(0.7)$ & $4(0.3)$ & $4(0.3)$ & $3(0.2)$ & $2(0.1)$ & $8(0.5)$ \\
\hline Cardiovascular death & $1(<0.1)$ & 0 & $2(0.2)$ & 0 & 0 & $1(0.1)$ & NA & NA \\
\hline Ischemic stroke & $1(<0.1)$ & $3(0.1)$ & $2(0.2)$ & $1(<0.1)$ & $3(0.2)$ & 0 & NA & NA \\
\hline Myocardial infarction & $3(0.1)$ & $6(0.3)$ & $4(0.3)$ & $3(0.2)$ & $1(0.1)$ & $2(0.2)$ & NA & NA \\
\hline
\end{tabular}

Figures in parentheses are percentages. NA $=$ Not available. ${ }^{1}$ During the on-treatment period.

amounts of blood transfused were similar in the two treatment groups (table 3).

\section{RECORD 2 Trial [29]}

In this study, 2,509 patients scheduled to undergo elective THR were assigned to receive oral rivaroxaban $10 \mathrm{mg}$ once daily $(\mathrm{n}=1,252)$ for $31-39$ days beginning 6-8 h after wound closure (patients also received placebo injections for 10-14 days, starting $12 \mathrm{~h}$ before surgery) or enoxaparin $40 \mathrm{mg}$ once daily subcutaneously $(\mathrm{n}=1,257)$ starting $12 \mathrm{~h}$ before surgery and restarting 6-8 h after wound closure, for 10-14 days (patients also received placebo tablets for 31-39 days starting 6-8 h after wound closure).

The primary efficacy outcome occurred in significantly fewer patients receiving extended thromboprophylaxis with rivaroxaban $(17 / 864$ patients $=2.0 \%)$ than in those receiving short-term enoxaparin (81/869 patients $=9.3 \%, p<0.0001)$, demonstrating the superiority of the treatment with rivaroxaban (fig. 2). Similarly, major VTE occurred in significantly fewer patients receiving extended thromboprophylaxis with rivaroxaban $(6 / 961$ patients $=0.6 \%)$ than in those receiving short-term prophylaxis $(49 / 962$ patients $=5.1 \%)$, with a difference statistically significant in favor of rivaroxaban $(\mathrm{p}<$ 0.0001). Furthermore, the observed rate of symptomatic VTE during the active study period was significantly lower in patients receiving rivaroxaban than in those receiving enoxaparin ( 0.2 vs. $1.2 \%, \mathrm{p}=0.004)$, while that found during the follow-up was similar in the two groups.
During the treatment period there were 2 deaths in the rivaroxaban group judged to have a cardiovascular cause, and 6 in the enoxaparin group judged as follows: 1 death related to PE, 4 unrelated to VTE, and 1 unexplained. During the follow-up period, 2 patients in the enoxaparin plus placebo group died (1 was judged to be related to PE and the other was unexplained).

The incidence of adverse events was similar in patients receiving extended thromboprophylaxis with rivaroxaban and short-term enoxaparin plus placebo (table 3). In particular, major bleeding occurred in 1 patient in each group, and hemorrhagic wound complications, requirements for drainage and blood transfusions and the amount of blood transfused were superimposable in each group.

An on-treatment increase in the plasma alanine aminotransferase level (i.e., a level of more than three times the upper limit of the normal range) occurred in $1.6 \%$ in the rivaroxaban group and in $4.7 \%$ in the enoxaparin group, with all cases resolving by the end of the follow-up period, apart from one in the enoxaparin group who had cholecystitis (no other follow-up data were available).

\section{RECORD 3 Trial [30]}

In this study, 2,531 patients who were to undergo TKR were assigned to receive for 10-14 days either oral rivaroxaban (10 mg once daily, $\mathrm{n}=1,254)$, beginning $6-8 \mathrm{~h}$ after wound closure, or subcutaneous enoxaparin (40 mg once daily, $\mathrm{n}=1,277)$, starting $12 \mathrm{~h}$ before surgery and restarting $6-8 \mathrm{~h}$ after wound closure. 
The primary efficacy outcome was significantly lower in the rivaroxaban group $(79 / 824$ patients $=9.6 \%)$ than in the enoxaparin group $(166 / 878$ patients $=18.9 \%, \mathrm{p}<$ 0.001 ) (fig. 2). Major VTE occurred in $1.0 \%$ of patients $(9 / 908)$ in the rivaroxaban group and in $2.6 \%$ of patients (24/925) in the enoxaparin group, with a statistically significant difference $(\mathrm{p}=0.01)$. The incidence of symptomatic VTE was significantly lower in the rivaroxaban group $(8 / 1,201$ patients $=0.7 \%)$ than in the enoxaparin group $(24 / 1,217$ patients $=2.0 \%, \mathrm{p}=0.005)$. The incidence of symptomatic VTE during the follow-up period was similar in the two groups (rivaroxaban $0.4 \%$ and enoxaparin $0.2 \%)$.

During the treatment period, there were no deaths or known PE in the rivaroxaban group and 2 unexplained deaths and 4 known PE in the enoxaparin group; during the follow-up period, there were 4 unexplained deaths in the enoxaparin group.

The incidence of adverse events was similar in patients receiving extended thromboprophylaxis with rivaroxaban and short-term enoxaparin plus placebo: major bleeding occurred in 1 patient in each group, and hemorrhagic wound complications, requirements for drainage and blood transfusions and the amount of blood transfused were superimposable in each group (table 3 ).

The on-treatment elevation in the plasma alanine aminotransferase level (i.e., a level of more than three times the upper limit of the normal range) was similar in the rivaroxaban and in the enoxaparin group (i.e. 1.7\%), with all cases resolving by the end of the follow-up period.

\section{RECORD 4 Trial [31]}

In this study, 3,148 patients scheduled for elective TKR were assigned to receive oral rivaroxaban $(10 \mathrm{mg}$ once daily) staring 6-8 h after wound closure or enoxaparin (30 mg twice daily, as approved in the US for the thromboprophylaxis of VTE) starting 12-14 h after wound closure.

The primary efficacy endpoint was significantly reduced in the rivaroxaban group (6.9 vs. $10.1 \%, \mathrm{p}=0.012$ ) (fig. 2), while the statistical significance was not reached for the other endpoints.

The incidence of bleeding events was similar in the two treatment groups, as was the safety profile concerning liver toxicity (table 3 ).

\section{Analysis of the RECORD 1-4 Trials}

A pooled analysis of data from the RECORD 1-3 trials [32] showed that in 9,581 patients rivaroxaban was more effective than enoxaparin in reducing the incidence of the composite of symptomatic VTE and all-cause mortality at 2 weeks ( 0.4 vs. $0.8 \%$, respectively; odds ratio 0.44 ; 95\% CI 0.23-0.79; $\mathrm{p}=0.005$ ) and at the end of the planned medication period ( 0.5 vs. $1.3 \%$, respectively; odds ratio 0.38 ; $95 \%$ CI $0.22-0.62 ; \mathrm{p}<0.001)$. The rate of major bleeding was similar at 2 weeks $(0.2 \%$ for both) and at the end of the planned medication period (0.3 vs. $0.2 \%)$.

At the time of this review, a pooled analysis of the RECORD studies had been presented at the last congress of the American Society of Hematology, but has not been published yet; so far, it has only been briefly summarized [33]. This prespecified pooled analysis was performed on all randomized patients who received at least one dose of double-blind study medication to evaluate the effect of rivaroxaban on the composite of symptomatic VTE (comprising DVT or PE) and death, and bleeding. These primary outcomes were analyzed at day $12 \pm 2$ in the active treatment pool (i.e. during the enoxaparin-controlled period common to all studies, to allow for unbiased comparison with enoxaparin) and for the total study duration pool (planned treatment period and 30-35 days followup). Rivaroxaban significantly reduced the incidence of symptomatic VTE and death compared with enoxaparin regimens at day $12 \pm 2$ (0.47 vs. $0.97 \% ; p=0.001)$ and for the total study duration ( 0.81 vs. $1.63 \% ; \mathrm{p}<0.001)$. Rivaroxaban was not associated with a statistically significant increased risk of major bleeding. These data demonstrate that in the regimens tested, rivaroxaban reduced the composite of major clinical outcomes compared with enoxaparin regimens, with no significant increase in the risk of major bleeding in patients undergoing major orthopedic surgery. So far, rivaroxaban seems to be more efficient than enoxaparin in preventing VTE in major orthopedic surgery, with the same safety profile but easier to handle [34].

The excellent results obtained in the RECORD trials and in phase I and II studies on rivaroxaban are of clinical relevance because generalizations can be made from the studied populations to daily clinical practice. As a matter of fact, rivaroxaban has several characteristics which make its use very attractive for the orthopedic surgeons, including: (1) The possibility to be administered orally at a fixed dose without need of laboratory monitoring. (2) A very low interference with food and several drugs (i.e. anti-acid, ranitidine, aspirin, naproxen, digoxin). (3) A favorable safety and efficacy profile also in special populations (elderly, obese, renally and hepatically impaired). In fact, age and gender do not influence efficacy and safety of the fixed dose of rivaroxaban, and this drug can also be used at the standard dose in extremely 
obese patients. Moreover, the dose of rivaroxaban does not have to be reduced in the presence of mild and moderate renal impairment (creatinine clearance $30-80 \mathrm{ml} /$ min). Limited clinical data for patients with severe renal impairment (creatinine clearance $15-29 \mathrm{ml} / \mathrm{min}$ ) indicate that rivaroxaban plasma concentrations are significantly increased in this patient population; therefore, rivaroxaban has to be used with caution in these patients. Finally, rivaroxaban is not recommended in patients with creatinine clearance $<15 \mathrm{ml} / \mathrm{min}$. Rivaroxaban is contraindicated in patients with hepatic disease associated with coagulopathy and clinically relevant bleeding risk, and it may be used with caution in cirrhotic patients with moderate hepatic impairment without coagulopathy. No dose adjustment is necessary in patients with other hepatic diseases. (4) In case of epidural analgesia, an epidural catheter should not be removed earlier than $18 \mathrm{~h}$ after the last administration of rivaroxaban, and the next dose should be administered no earlier than $6 \mathrm{~h}$ after removal of the catheter [32].

On the other hand, specific studies should assess the optimal strategy for a reversal of the anticoagulant effect of rivaroxaban; at this time, although there is no specific antidote, preclinical studies suggest that recombinant factor VIIa [35] or activated prothrombin complex (FEIBA) [36] may reverse the effects of a high dose of rivaroxaban. If strategies such as delayed administration or discontinuation of the anticoagulant drug, mechanical compression, surgical intervention, and blood product transfusion fail to control bleeding, the administration of recombinant factor VIIa or FEIBA should be considered.

\section{Apixaban}

Apixaban (BMS-562247) is a follow-up compound of razaxaban and is thought to have superior pharmacologic properties in particular with respect to bleeding. It is a small molecule able to inhibit in a selective and reversible manner the active site of both free and prothrombinasebound factor Xa $[37,38]$.

Preclinical studies demonstrated that apixaban has an oral bioavailability of 50\%: it is absorbed in the gastrointestinal tract, its plasma peak is reached in about $3 \mathrm{~h}$ and its half-life is about $12 \mathrm{~h} \mathrm{[38].} \mathrm{Food} \mathrm{does} \mathrm{not} \mathrm{interfere} \mathrm{with}$ its absorption so that the drug generates a predictable anticoagulation effect. Apixaban is metabolized in the liver by CYP-dependent and CYP-independent mechanisms, and approximately $25 \%$ is cleared through the renal route whereas the remainder appears in the feces [39].

Oral Factor Xa Inhibitors for VTE

Prevention in Major Orthopedic Surgery
To date, the results of one phase II study have been published. The APROPOS study (acronym for Apixaban Prophylaxis in Patients Undergoing Total Knee Replacement Surgery) [40] randomized 1,238 patients to test the efficacy and the safety of three total daily doses of 5, 10 and $20 \mathrm{mg}$ of apixaban given as once or twice daily doses following elective TKR. The comparators were enoxaparin $30 \mathrm{mg}$ twice daily or warfarin titrated to an INR of 1.8-3.0. The study showed that all apixaban dose groups had point estimates for primary efficacy outcome (composite of adjudicated VTE events - including asymptomatic and symptomatic DVT and symptomatic nonfatal PE - and death from any cause) lower than both the enoxaparin and warfarin groups, with a relative risk reduction of 21-69 and 53-82\%, respectively. There was a clear dose effect on total bleeding rates, with superimposable rates between treatments when comparing $5 \mathrm{mg}$ total daily dose of apixaban (3.9\%) versus enoxaparin (4.0\%) or versus warfarin (5.3\%). The incidence of an increase in liver enzymes was similar in all treatment groups. As far as the authors found lower VTE rates among patients treated with twice versus once daily dosing, they state that $2.5 \mathrm{mg}$ twice daily is the preferred regimen.

Four phase III studies have evaluated apixaban for VTE prevention in major or thopedic surgery.

The ADVANCE-1 clinical trial was a phase III randomized double-blind multicenter study that evaluated the efficacy and safety of apixaban, an oral direct factor Xa inhibitor, $2.5 \mathrm{mg}$ orally twice daily compared with enoxaparin $30 \mathrm{mg}$ subcutaneously every $12 \mathrm{~h}$ for preventing VTE after TKR. At the time of this review, the results of the ADVANCE-1 study had been presented at the last congress of the American Society of Hematology, but have not been published yet; so far, they have only been briefly summarized [41]. Apixaban (or oral placebo) and enoxaparin (or subcutaneous placebo) were started 12$24 \mathrm{~h}$ postoperatively (mean $20 \mathrm{~h}$ ) and continued until mandatory bilateral venography was completed at $12 \pm$ 2 days. The primary efficacy outcome was the composite of DVT by venography, symptomatic, objectively confirmed DVT or PE, or death from any cause during the treatment period. The secondary efficacy outcome was the composite of objectively confirmed proximal DVT or $\mathrm{PE}$, or death. The primary safety outcome was bleeding, including major bleeding, clinically relevant nonmajor bleeding, and minor bleeding. The study hypothesis was that apixaban would be as effective as enoxaparin based on a prespecified noninferiority margin in which the upper limit of the two-sided $95 \% \mathrm{CI}$ is $<1.25$ for relative risk and $<5.6 \%$ for the absolute risk difference for the prima- 
ry efficacy outcome. The primary efficacy outcome occurred in 104 of 1,157 patients (8.99\%) given apixaban and in 100 of 1,130 (8.85\%) given enoxaparin (relative risk $1.02,95 \%$ CI $0.78-1.32$, one-sided $p=0.064$ for noninferiority, statistical criteria not met). Major or clinically relevant nonmajor bleeding occurred in 46 of 1,596 patients $(2.88 \%)$ given apixaban compared with 68 patients (4.28\%) given enoxaparin (absolute difference $1.46 \%$, two-sided $\mathrm{p}=0.034)$. Major bleeding occurred in $11 \mathrm{pa}-$ tients $(0.69 \%)$ who received apixaban and in 22 patients $(1.39 \%)$ who received enoxaparin ( $t w o-$ sided $\mathrm{p}=0.053$ ).

One study has been completed, but the results are not available yet. The NCT00097357 is a phase II and phase III randomized, double-blinded (apixaban and enoxaparin), active-controlled (enoxaparin and warfarin), parallel-arm, dose-response study of the oral factor Xa inhibitor apixaban in subjects undergoing elective TKR surgery. This study was designed to evaluate whether apixaban can prevent blood clots in the legs and lungs after unilateral TKR surgery in comparison with enoxaparin and warfarin. The safety of this treatment will also be studied (www.clinicaltrials.gov).

For the other two studies, the recruitment is still ongoing. The NCT00423319 is a phase III randomized, double-blind, active-controlled, parallel-group, multicenter study to evaluate the safety and efficacy (prevention of DVT and PE) of apixaban in patients undergoing elective THR surgery. The safety of apixaban will also be studied (www.clinicaltrials.gov). The NCT00452530 is a phase III, randomized, double-blind, active-controlled (enoxaparin), parallel-group, multicenter study to evaluate the safety and efficacy (prevention of DVT and PE) of apixaban in subjects undergoing elective TKR surgery (ADVANCE-2 study) and to learn how apixaban compares to enoxaparin as regards preventing these clots. The safety of apixaban will also be studied (www.clinicaltrials.gov).

\section{LY517717}

This direct factor Xa inhibitor has an oral bioavailability of $25-82 \%$ [42]; because of its anticoagulant activity that peaked $0.5-4 \mathrm{~h}$ following oral administration and owing to its prolonged half-life of $25 \mathrm{~h}$ it could be administered once daily [43]. LY517717 is apparently primarily cleared via the gastrointestinal route [43].

The phase II study of LY517717 has recently been published [43]: it was a multicenter, randomized, double-blind, double-dummy, dose-escalation/parallel arm designed study to determine whether this drug can safely reduce the risk of VTE in patients undergoing TKR or THR, and to identify one or more dose regimens that are not inferior to enoxaparin. To verify this hypothesis, 511 patients were randomized to oral LY517717 25, 50, $75 \mathrm{mg}$ once daily or enoxaparin $40 \mathrm{mg}$ s.c. Higher doses of LY517717 (100, 125, $150 \mathrm{mg}$ ) were to be introduced if the lowest were insufficiently effective and did not cause excessive bleeding. The study demonstrated that LY517717 is an efficacious and safe oral antithrombotic agent that achieved a reduction in primary endpoint (composite of DVT detected by bilateral venography at the end of the study period, symptomatic DVT and/or PE during treatment) similarly to $40 \mathrm{mg}$ enoxaparin (21.2\%) at the dose of $100-150 \mathrm{mg}$ once daily (15.6-18.8\%) and a lower although not significant incidence of major (0.0-0.9 vs. $1.1 \%)$ and minor bleeding ( $0.0-$ 1.0 vs. $2.2 \%$ ). There were no statistically significant differences between treatment groups in the percentage of patients that experienced an elevation of liver enzymes.

At the time of this review, there are no ongoing or programmed phase II or phase III trials on LY517717.

\section{YM150}

This drug is a potent and specific orally active, direct inhibitor of factor Xa. Phase I studies demonstrated that YM150 is well tolerated with predictable pharmacokinetics and pharmacodynamics in healthy young and elderly volunteers [43]. Moreover, food does not interfere with the absorption of the drug, and its antithrombotic action is almost immediate [43].

A phase II study was designed as a randomized, controlled trial to assess safety and efficacy of YM150 in a wide dose range in comparison with enoxaparin in patients undergoing primary THR surgery. To this purpose, 178 patients were randomized to receive $3,10,30$ and 60 $\mathrm{mg}$ once daily of YM150 or $40 \mathrm{mg}$ s.c. of enoxaparin. The primary endpoint (i.e. the occurrence of a major and/or clinically relevant nonmajor bleeding event) was similar in the enoxaparin (22.2\%) and YM150 (11.8-23.5\%) groups at any dose. The main efficacy outcome (composite endpoint of DVT detected by bilateral venography at the end of the study period, symptomatic DVT or nonfatal PE and/or death) was evaluated as VTE rate, as long as it had been the only thromboembolic event that occurred. The VTE incidence was reduced by increasing YM150 doses with a significant dose-related trend ( $\mathrm{p}=$ 0.006). The VTE rate in the YM150 group ranged from $51.9 \%$ in the $3-\mathrm{mg}$ group to $18.5 \%$ in the $60-\mathrm{mg}$ group, 
while the VTE incidence in the enoxaparin group was $38.7 \%$. There was no evidence of liver injury. This study did not determine the optimum dosage of YM150.

To date, two studies are ongoing: NCT00408239 is a phase II randomized, enoxaparin-controlled, open-label, dose-escalation study in which the safety and tolerability of escalating oral doses of YM150 for the prevention of VTE in patients undergoing elective TKR are evaluated; NCT00595426 is a phase IIb, double-blind, double-dummy, randomized, parallel-group study to evaluate the safety and efficacy of twice daily dosing and once daily dosing of YM150 in subjects undergoing primary elective TKR in comparison with warfarin.

YM150 seems to be a promising new oral factor Xa inhibitor, owing to its demonstrated efficacy and safety in preventing VTE in hip and knee replacement surgery.

\section{Betrixaban}

Betrixaban is an oral direct inhibitor of factor $\mathrm{Xa}$, which has been developed for the prevention of VTE.

An exploratory randomized, controlled clinical trial was designed to assess safety and efficacy of betrixaban in a wide dose range in comparison with enoxaparin, in patients undergoing elective TKR surgery [44]. To this purpose, 215 patients were randomized in a $2: 2: 1$ ratio to receive postoperative oral betrixaban $15 \mathrm{mg}$ or $40 \mathrm{mg}$ once daily or $20 \mathrm{mg}$ s.c. every $12 \mathrm{~h}$ of enoxaparin, respectively, for 10-14 days. The incidence of the main efficacy outcome (composite endpoint of DVT detected by unilateral venography at the end of study period, symptomatic DVT and PE) was $14 / 70$ (20\%; 95\% CI 11-31) for betrixaban $15 \mathrm{mg}, 10 / 65$ (15\%; 95\% CI 8-27) for betrixaban 40 $\mathrm{mg}$, and $4 / 40(10 \%$; $95 \%$ CI $3-24)$ for enoxaparin. No bleeds were reported for betrixaban $15 \mathrm{mg}, 2$ (2.4\%) clinical significant nonmajor bleeds were reported with be- trixaban $40 \mathrm{mg}$, and 1 (2.3\%) major and $2(4.6 \%)$ clinical significant nonmajor bleeds for enoxaparin. In conclusion, betrixaban demonstrated antithrombotic activity and appeared to be well tolerated in knee replacement patients at the doses studied.

\section{Conclusions}

Since the discovery of anticoagulation therapy, heparins and coumarins have been the only available drugs, with all their well-known pros and cons. After over 60 years of monopoly, it seems that new classes of anticoagulants are becoming available. These new drugs share the important characteristic of inhibiting only one enzyme of the coagulation cascade and the selective inhibition of factor Xa seems very appealing. Fondaparinux has been the first molecule of this new class to reach the market: it is an indirect inhibitor of factor Xa, but it still requires parenteral administration. Rivaroxaban, apixaban, LY517717, YM150 and betrixaban are all oral direct inhibitors of factor $\mathrm{Xa}$ and represent the most investigated new anticoagulants for the prevention of VTE in major orthopedic surgery. Rivaroxaban is currently the furthest ahead in its developing program and has recently been approved for clinical use just for this indication.

In summary, oral factor Xa inhibitors are undoubtedly a further improvement in anticoagulation therapy and appear to be a very promising strategy for thromboprophylaxis in orthopedic surgery. Future studies should assess their cost-effectiveness compared with the currently available anticoagulant drugs in daily clinical practice as well as the optimal strategy for reversal of their anticoagulant effect. Finally, ongoing surveillance of efficacy and safety of oral factor Xa inhibitors in special populations (elderly, obese, underweight, renally and hepatically impaired) is also warranted.

\section{References}

\footnotetext{
$\nabla_{1}$ Silverstein MD, Heit JA, Mohr DN, et al: Trends in the incidence of deep vein thrombosis and pulmonary embolism: a 25-year population-based study. Arch Intern Med 1998;158:585-593.

-2 Ageno W, Squizzato A, Garcia D, Imberti D: Epidemiology and risk factors of venous thromboembolism. Semin Thromb Hemost 2006;32:651-658.

3 Cohen AT, Agnelli G, Anderson FA, et al: Venous thromboembolism (VTE) in Europe. The number of VTE events and associated morbidity and mortality. Thromb Haemost 2007;98:756-764.
}

\footnotetext{
4 Geerts WH, Pineo GF, Heit JA, et al: Prevention of venous thromboembolism: the Seventh ACCP Conference on Antithrombotic and Thrombolytic Therapy. Chest 2004;126: 338S-400S.

5 Pellegrini VD, Donaldson CT, Farber DC, et al: Prevention of readmission for venous thromboembolic disease after total hip arthroplasty. Clin Orthop 2005;441:56-62.

-6 Pellegrini VD, Donaldson CT, Farber DC, et al: The Mark Coventry Award: prevention of readmission for venous thromboembolism after total knee arthroplasty. Clin Orthop 2006;452:21-27.
}

Oral Factor Xa Inhibitors for VTE

Prevention in Major Orthopedic Surgery
Pathophysiol Haemost Thromb 2007-08;36:217-226 
$\checkmark 7$ Geerts WH, Bergqvist D, Pineo GM, et al: Prevention of venous thromboembolism. American College of Chest Physicians Evidence-Based Clinical Practice Guidelines (8th edition). Chest 2008;133:381S-453S.

8 Weitz JI, Hirsh J, Samama MM: New antithrombotic drug. American College of Chest Physicians Evidence-Based Clinical Practice Guidelines (8th edition). Chest 2008;133: 234S-256S.

-9 Turpie AG, Bauer KA, Eriksson BI, et al: Superiority of fondaparinux over enoxaparin in preventing venous thromboembolism in major orthopedic surgery using different efficacy end points. Chest 2004;126:501-508.

$\checkmark 10$ Warkentin TE, Greinacher A, Koster A, et al: Treatment and prevention of heparininduced thrombocytopenia. American College of Chest Physicians Evidence-Based Clinical Practice Guidelines (8th edition). Chest 2008; 133:330S-380S.

-11 Furie B, Furie BC: Molecular basis of vitamin K-dependent $\gamma$-carboxylation. Blood 1990; 75:1753-1762.

$\checkmark 12$ Harenberg J: Indirect and direct anticoagulants predominantly inhibiting factor Xa. Therapy 2008;5:177-192.

13 Bauer KA: New anticoagulants: anti IIa vs anti Xa - is one better? J Thromb Thrombolysis 2006;21:67-72.

-14 Turpie AG: Oral, direct factor Xa inhibitors in development for the prevention and treatment of thromboembolic diseases. Arterioscler Thromb Vasc Biol 2007;27:1238-1247.

-15 Strassburger J, Wilmen A, Pohlmann J, et al: In vitro and in vivo studies of the novel antithrombotic agent BAY 59-7939 - an oral, direct factor Xa inhibitor. J Thromb Haemost 2005;3:514-521.

- 16 Kubitza D, Becka M, Wensing G, et al: Safety, pharmacodynamics, and pharmacokinetics of BAY 59-7939 - an oral, direct factor Xa inhibitor - after multiple dosing in healthy male subjects. Eur J Clin Pharmacol 2005;61: 873-880.

17 Gross PL, Weitz JI: New anticoagulants for treatment of venous thromboembolism. Arterioscler Thromb Vasc Biol 2008;28:380386.

$\checkmark 18$ Kubitza D, Becka M, Zuehlsdorf M, et al: Body weight has limited influence on the safety, tolerability, pharmacokinetics, or pharmacodynamics of rivaroxaban (BAY 59-7939) in healthy subjects. J Clin Pharmacol 2007;47:218-226.

19 Kubitza D, Becka M, Mueck W, et al: The effect of extreme age, and gender, on the pharmacology and tolerability of rivaroxaban, an oral, direct factor Xa inhibitor (abstract). Blood 2006;108:905.

20 Halabi A, Maatouk H, Klause N, et al: Effects of renal impairment on the pharmacology of rivaroxaban (BAY 59-7939), an oral, direct, factor Xa inhibitor (abstract). Blood 2006; 108:913.
21 Kubitza D, Becka M, Zuehlsdorf M, et al: Effect of food, an antacid, and the $\mathrm{H} 2$ antagonist ranitidine on the absorption of BAY 597939 (rivaroxaban), an oral, direct factor Xa inhibitor, in healthy subjects. J Clin Pharmacol 2006;46:549-558.

22 Kubitza D, Becka M, Mueck W, et al: Safety, tolerability, pharmacodynamics, and pharmacokinetics of rivaroxaban - an oral, direct factor Xa inhibitor - are not affected by aspirin. J Clin Pharmacol 2006;46:981-990.

23 Turpie A, Fisher W, Bauer K, et al: BAY 597939: an oral, direct factor Xa inhibitor for the prevention of venous thromboembolism in patients after total knee replacement: a phase II dose-ranging study. J Thromb Haemost 2005;3:2479-2486.

24 Eriksson BI, Borris LC, Dahl OE, et al: Oral, direct factor Xa inhibition with BAY 59-7939 for the prevention of venous thromboembolism after total hip replacement. J Thromb Haemost 2006;4:121-128.

25 Eriksson BI, Borris LC, Dahl OE, et al: A once-daily, oral, direct factor Xa inhibitor, rivaroxaban (BAY 59-7939), for thromboprophylaxis after total hip replacement. Circulation 2006;114:2374-2381.

26 Eriksson BI, Borris LC, Dahl OE, et al: Doseescalation study of rivaroxaban (BAY 597939) - an oral, direct factor Xa inhibitor - for the prevention of venous thromboembolism in patients undergoing total hip replacement. Thromb Res 2007;120:685-693.

27 Mueck W, Borris LC, Dahl OE, et al: Population pharmacokinetics and pharmacodynamics of once- and twice-daily rivaroxaban for the prevention of venous thromboembolism in patients undergoing total hip replacement. Thromb Haemost 2008; 100:453-461.

28 Eriksson BI, Borris LC, Friedman RJ, et al: Rivaroxaban versus enoxaparin for thromboprophylaxis after hip arthroplasty. N Engl J Med 2008;358:2765-2775.

29 Kakkar AK, Brenner B, Dahl OE, et al: Extended duration rivaroxaban versus shortterm enoxaparin for the prevention of venous thromboembolism after total hip arthroplasty: a double-blind, randomized controlled trial. Lancet 2008;372:31-39.

30 Lassen MR, Ageno W, Borris LC, et al: Rivaroxaban versus enoxaparin for thromboprophylaxis after total knee arthroplasty. N Engl J Med 2008;358:2776-2786.

-31 Turpie AG, Lassen MR, Davidson BL, et al: Rivaroxaban versus enoxaparin for thromboprophylaxis after total knee arthroplasty (RECORD4): a randomised trial. Lancet 2009;373:1673-1680.

-32 Eriksson BI, Kakkar AK, Turpie AG, et al: Oral rivaroxaban for the prevention of symptomatic venous thromboembolism after elective hip and knee replacement. J Bone Joint Surg Br 2009;91:636-644.
33 Turpie A, Lassen M, Kakkar AJ, et al: A pooled analysis of four pivotal studies of rivaroxaban for the prevention of venous thromboembolism after orthopaedic surgery: effect on symptomatic venous thromboembolism, death and bleeding (abstract 36). American Society of Hematology 2008 Annual Meeting, San Francisco, 2008.

34 Imberti D, Dall'Asta C: Clinical benefits of rivaroxaban for thromboprophylaxis after major orthopaedic surgery. Adv Orthop 2008;1:2-8.

35 Gruber A, Marzec UM, Buetehorn U, et al: Recombinant factor VIIa reverses the effects of a high dose of rivaroxaban in baboons (abstract). Pathophysiol Haemost Thromb 2007; 36(suppl 1):A39.

36 Perzborn E, Tinel H: FEIBA reverses the effects of a high dose of rivaroxaban in rats (abstract). Pathophysiol Haemost Thromb 2007; 36(suppl 1):A40.

37 Pinto DJ, Orwat MJ, Koch S, et al: Discovery of 1-(4-methoxyphenyl)7-oxo-6-(4-(2-oxopiperidin-1-yl)phenyl)-4,5,6,7-tetrahydro$1 \mathrm{H}$-pyrazolo[3,4-c]pyridine-3-carboxamide (apixaban, BMS-562247), a highly potent, selective, efficacious, and orally bioavailable inhibitor of blood coagulation factor Xa. J Med Chem 2007;50:5339-5356.

-38 Wong PC, Crain EJ, Xin B, et al: Apixaban, an oral, direct and selective factor Xa inhibitor: in vitro, antithrombotic and antihemostatic studies. J Thromb Haemost 2007;6: 820-829.

39 Raghavan N, Frost CE, Yu Z, et al: Apixaban metabolism and pharmacokinetics after oral administration to humans. Drug Metab Dispos 2009;37:74-81.

40 Lassen MR, Davidson BL, Gallus A, et al: The efficacy of apixaban, an oral, direct factor Xa inhibitor, as thromboprophylaxis in patients following total knee replacement. J Thromb Haemost 2007;5:2368-2375.

41 Lassen M, Gallus A, Pineo G, et al: Randomized double-blind comparison of apixaban with enoxaparin for thromboprophyaxis after knee replacement: the ADVANCE-1 trial (abstract 31). American Society of Hematology 2008 Annual Meeting, San Francisco, 2008.

42 Agnelli G, Haas SK, Krueger KA: A phase II study of the oral factor Xa inhibitor LY517717 for prevention of venous thromboembolism after hip or knee replacement. J Thromb Haemost 2007;5:746-753.

43 Eriksson BI, Turpie AGG, Lassen MR, et al: A dose escalation study of YM150, an oral direct factor $\mathrm{Xa}$ inhibitor, in the prevention of venous thromboembolism in elective primary hip replacement. J Thromb Haemost 2007;5:1660-1665.

44 Turpie AG, Bauer KA, Davidson BL, Fisher WD, Gent M, Huo MH, Sinha U, Gretler DD, EXPERT Study Group: A randomized evaluation of betrixaban, an oral factor Xa inhibitor, for prevention of thromboembolic events after total knee replacement (EXPERT). Thromb Haemost 2009;101:68-76. 